\title{
Multi-user Expert System for Operation and Mainte- nance in Energized Lines
}

\author{
Erika F. Moreno, Evelyn E. Pacheco, Víctor H. Andaluz and Álvaro S. Mullo. \\ Universidad de las Fuerzas Armadas ESPE, Sangolquí - Ecuador \\ \{efmorenol, eepachecol, vhandaluz1, asmullo\}@espe.edu.ec
}

\begin{abstract}
The article presents the development of a multi-user application in virtual reality for the training and theoretical-practical training of electrical maintenance personnel in the operation of energized lines. The environment has a safety explanatory room in which users will know each of the rules and protocols applied to perform maintenance maneuvers and operation of energized lines, a realistic work area of the electrical power system that starts from hydroelectric generation and passes through transmission lines, subtransmission to reach substations that raise or reduce the voltage for subsequent distribution. The environment has been created using photogrammetry techniques, WorldComposer, CAD design tools, Unity 3D, DigSilent Power Factory and Matlab to provide realism of electrical system behavior, emulating failures and critical conditions caused by external and internal emergency events. Experimental tests show the efficiency of the system generated by human-machine interaction in which operators interact between themselves and the ambience, facilitate immersion in an environment that offers to contribute to the development of their risk-free collaborative skills and abilities.
\end{abstract}

Keywords: Virtual Reality, Maintenance on Energized Lines, Electrical Power System, Photogrammetry, Unity 3D.

Palabras clave: Realidad Virtual, Mantenimiento de Líneas Energizadas, Sistema Eléctrico de Potencia, Fotogrametría, Unity 3D.

\section{Introduction}

Maintenance in industrial systems is a strategic factor to guarantee a high level of safety and productivity [1]. The development of adequate maintenance policies guarantees the efficiency of production plants in terms of quality to minimize costs and maximize the availability and performance of fixed assets [2]. Several maintenance policies have been introduced, such as fault-based (FBM), usage-based maintenance (UBM), condition-based maintenance (CBM), design and output maintenance (DOM) and detectionbased maintenance. Ideally, maintenance should be performed shortly before the asset fails, not too soon, but not too late either [1] [2]. The most common practice in the industry is preventive maintenance; a piece of equipment is regularly serviced while in operation so that it does not cause unplanned downtime. Predictive maintenance, on the 
other hand, uses advanced asset-based analysis under real operating conditions [3]. Specifically in the electric power industry has moved to a competitive and intelligent future, with the need to improve maintenance management tasks [4].

Electric utilities apply proactive methods based on RCAM, by improving maintenance modeling that includes details of the procedure to the transmission system. It is divided into components and subcomponents, using weighting coefficients for prioritization [4], in order to maintain continuity of electricity and stable supply. The appropriate procedure for transmission lines and protection systems is the preventive one [4]. When developing maintenance operations in energized electrical networks, the proximity between the liniero and the energized conductor requires the implementation of several shielding procedures. Shielding against the electric field is provided by the use of dielectrically shielded equipment, being highly effective as the low frequency electric field is relatively easy to protect [5]. It is essential to follow an effective overhead power line safety program, in which OSHA establishes as regulation, specific distances and requirements for this type of work and equipment, as well as for workers, OSHA1926, Subpart V power transmission and distribution; ANSI IEEE C2- National Electrical Safety Code (NESC), 1910.333(c) for work near exposed energized parts, and NFPA 70E2015 Standard for Electrical Safety in the workplace, Section 130.8 establishes equipment requirements [6]. Line operators and electricians must coordinate their senses and actions under the practice of proper and safe work as a team. Therefore, operator learning and training is becoming a strategic task for utilities that include immersive technologies, with training systems that can be integrated into the work environment and adaptive training methods to improve different levels[7].

Virtual Reality (VR) and Augmented Reality (AR) systems tend to be a safer solution in terms of training methods, in which various procedures can be simulated by providing representations of the real world with dangerous environments without risking people and equipment [7] [8]. Most power system training applications are associated with hazardous tasks. Within the training processes, several research projects are mentioned, such as: $i$ ) Implementation of a training program based on VR, applied to the maintenance of medium voltage overhead lines in electricity distribution networks, the system has allowed a substantial reduction in electric accident [9]; $i$ ) Desktop (VR) environment for power line workers, the training system aims to reinforce classroom training and bridge theoretical knowledge and field work training [10]; iii) Interactive system of virtual training of maintenance of hydroelectric generation equipment to improve the experience and effectiveness of the training, based on the operating system of each part and component [11]; iv) Architecture of an intelligent training system based on virtual environments for electricity distribution substations oriented to the training process of electricians in the area of electrical substations [12]; v) An immersive Virtual Reality application for collaborative training of power system operators to improve user immersion and a problem-based learning approach [13]; vi) Training in virtual environments for a hybrid plant oriented to the simulation of faults and manoeuvres for the training of professionals in Electrical Systems [14]. 
The document presents the development of a training system in VR oriented to the operation and maintenance in energized lines of a real environment from the generation, transmission, subtransmission and distribution of electric energy, in which the personnel according to the hierarchical level can access the application and be trained in an immersive environment free of risks. The realism of the application is established from an electrical scheme allowing the simulation and analysis of faults occurring in the bars of the electric power system, as well as the variation of the dielectric characteristics of the safety equipment itself that will improve the skills, skills of operators and collaborative work.

This work is divided into 5 sections, including the introduction. Section 2 describes the structure of the proposed system. Section 3 includes the description of the virtual environment. The development of collaborative work using the multi-user system is described in section 4. Section 5 analyses the results obtained. Finally, section 6 presents the conclusions of the training system.

\section{System Structure}

In the electrical area is of vital importance group coordination between maintenance personnel due to the risk of working with high voltage, one of the concurrent problems is the low capacity for collaborative interaction of the operators. [15]. Some studies related to the development of virtual applications based on interactive learning allow to develop techniques of comprehensive learning of the construction team in the processes and operational safety. [16].

The development of an immersive multi-user expert system for operation and maintenance work on energized lines is presented, based on hierarchical levels according to the activities in charge.; i) chief of maintenance;ii) overseer; iii) chief of group y iv) operator, of the selection of the avatar depends on the tasks to be performed in the work area. The virtualization of the real environment is carried out through photogrammetry techniques and an additional tool to create height maps WorldComposer, while the simulation of the electrical power system is developed in the DigSilent Power Factory-Matlab software which provides realism and stabilizes the behavior of the electrical system in the event of a failure or external critical situation that leads to an excessive increase in voltage and current, directly affecting the elements of the system such as transformers, isolates, transmission lines, subtransmission, etc. in order to facilitate maintenance operators a learning and interaction with the system as real as possible and me-improve the skills for the detection of hazards. The architecture of the virtual training system is (shown in Fig. 1).

The environment has two training scenes; $i$ ) information room, training place on the mandatory use of individual protection equipment and maintenance protocols according to the maneuver to be carried out in subtransmission or distribution; ii) work area, presents a real environment of a power electrical system with the respective lifting and 
reducing substations, transmission lines, subtransmission and distribution with the main feeders, place to which operators will have access prior to training in the information room.

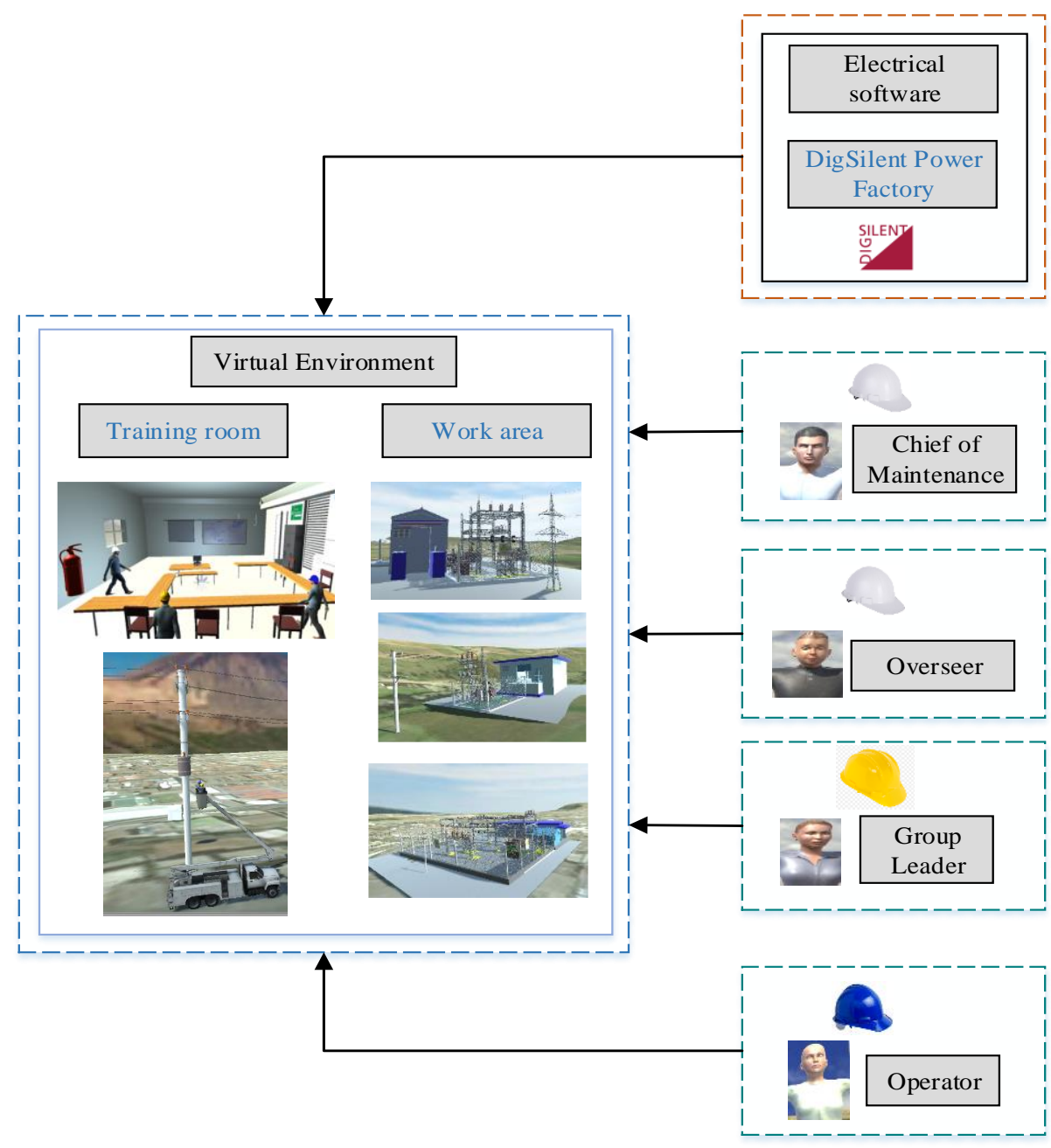

Fig. 1. Virtual Training System Architecture

\section{Virtual Environment}

This section describes the application development methodology.

The stages are; $i$ ) Creation of the environment, this stage is subdivided into: a) virtualization of the environment, with the objective of making the training process for the 
maintenance personnel of energized lines as real as possible; b) 3D Modeling, the information is collected by means of photographs and georeferencing for the construction of the scene; ii) Virtual environment, is developed in the Unity 3D graphics engine, incorporating features to the model CAD and the environment. DigSilent Power Factory generates process realism from critical system conditions. There are two scenes in the environment; a) Information room and b) Work area.iii) Multi-user, at this stage, a multi-user hierarchical system will be implemented so that operators from different parts of the world can connect to the environment through a communication network and interact with each other, strengthening collaborative work. The structural diagram of interrelation between components is (shown in Fig. 2).

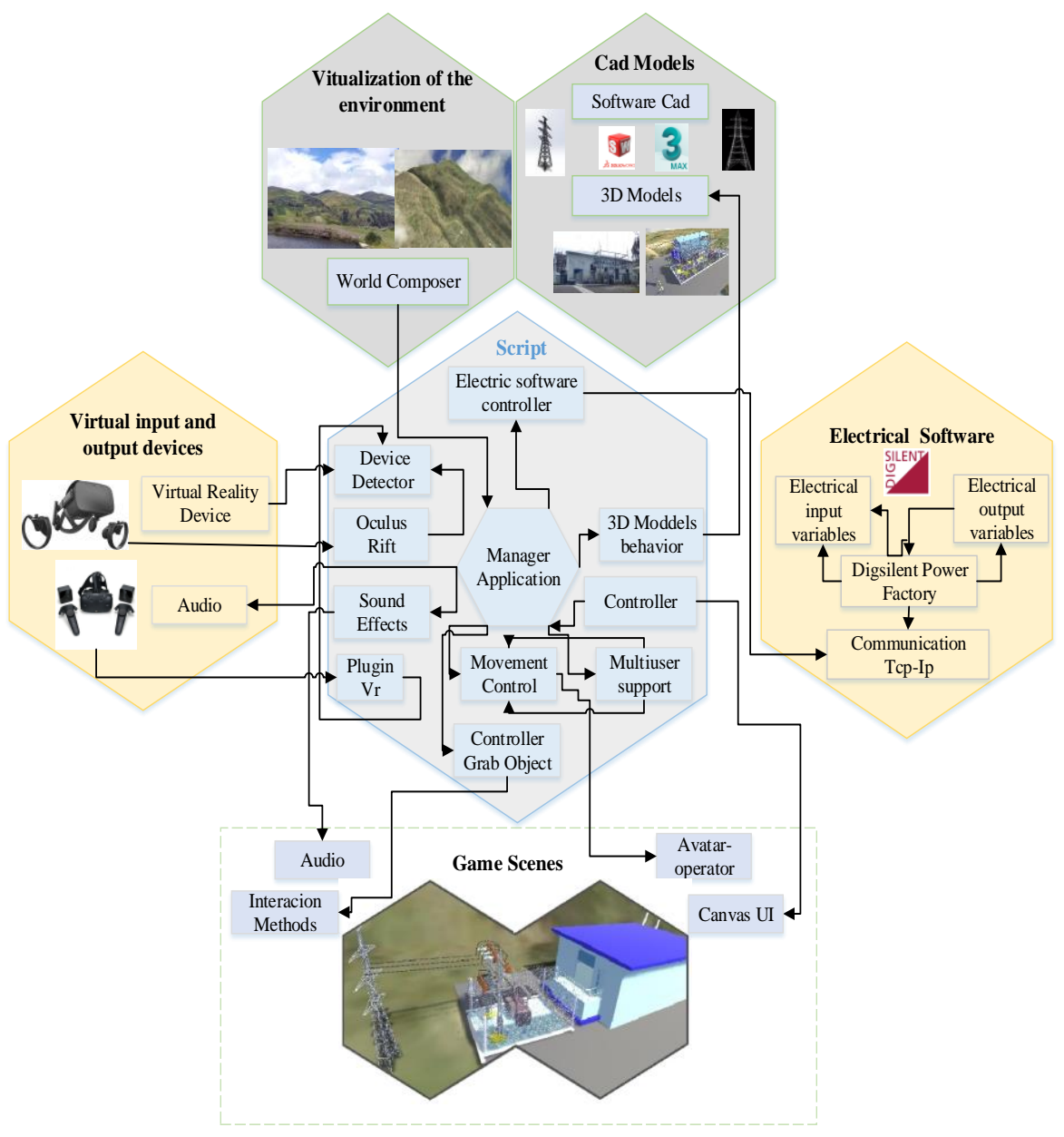

Fig 2. Structural diagram of interrelation between components 


\subsection{Virtualization of the environment}

The real environment to be virtualized is part of the National Interconnected System of Ecuador, specifically in Cotopaxi the Illuchi 1 - El Calvario subtransmission line, the Illuchi 1 hydroelectric power station has an elevation substation that is interconnected with the ELEPCO system through a three-phase line of $22 \mathrm{KV}$ to the $13.8 \mathrm{Kv}$ El Calvario line. (See Fig. 3).

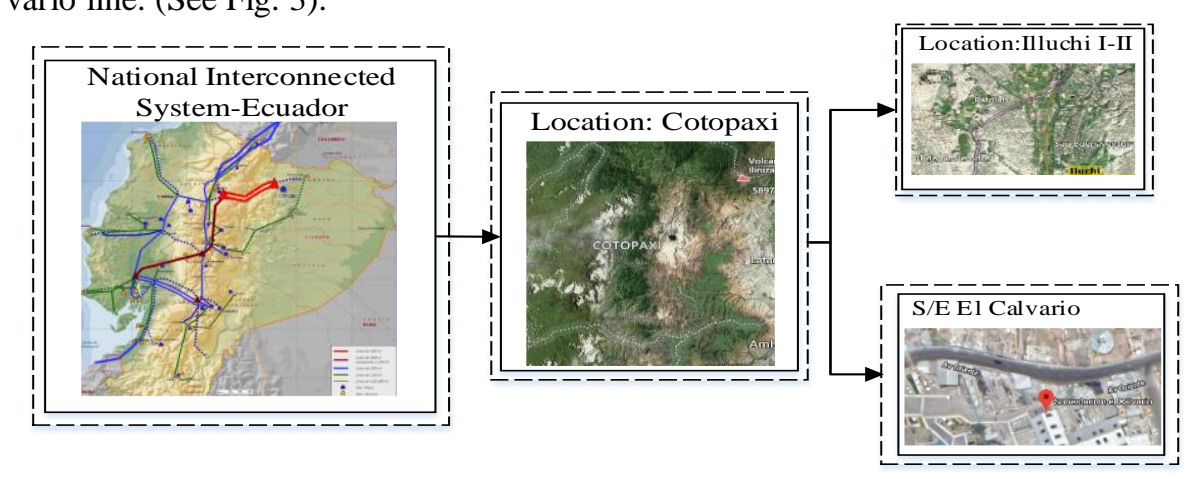

Fig. 3. Description of the environment

The creation of the environment is developed from the survey of information photographies and georeferencing, the tool used to extract data from real world maps is WorldComposer, for the exact location of the area is needed coordinates, latitude and longitude, facilitating the creation of reliefs in 3D. The description for the virtualization of the environment can be (seen in Fig. 4). 


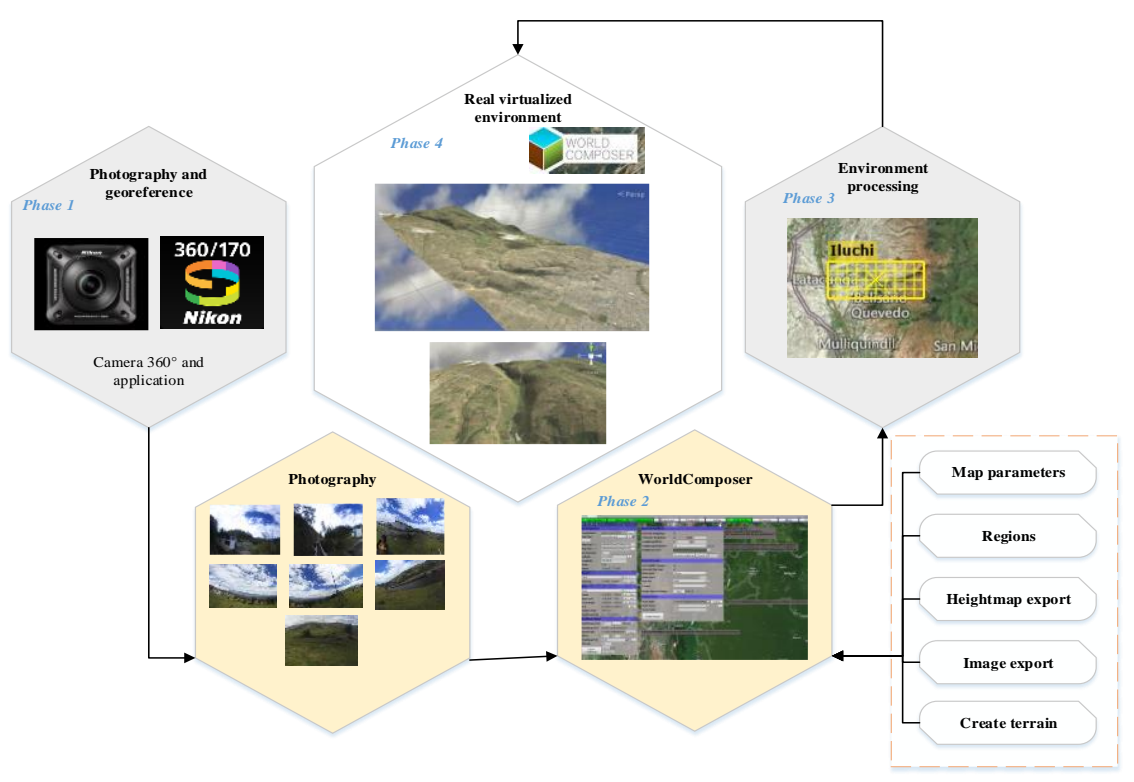

Fig. 4. Description of the virtualization of the environment

In the first phase it is required the survey of information of the exact place to be virtualized with the coordinates of location respectively, same that is obtained with photographs in $360^{\circ}$; each photograph has as data the georefencia-tion of the real point in which it was taken. The WorldComposer tool allows to extract height maps with images of a real location of any part of the world, for its initialization the icons described below must be activated: map parameters, regions, heightmap export, image export and create terrain, once activated the parameters the vir-tualized area is created directly in the Unity assets.

\subsection{CAD Design}

SolidWorks CAD modeling software facilitates the creation from design, import and assembly of subtransmission and distribution structures, posts, insulated-res and transformers of elevation and reduction substations to establish a scene as close to reality as possible. Each design needs to be saved with a *.IGS extension compatible with 3DSMax to import into Unity. The CAD design of certain elements is (shown in Fig. $5)$. 


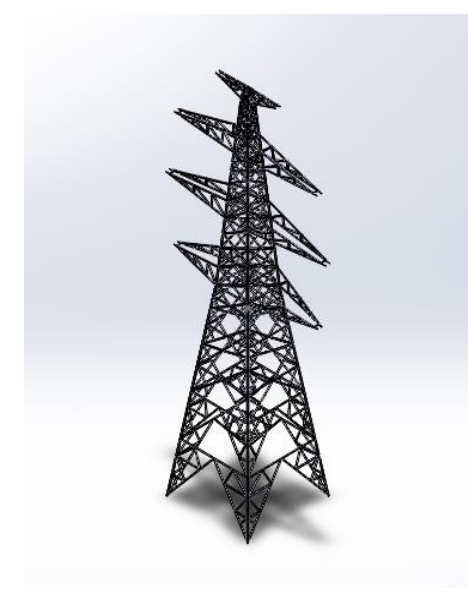

(a) Subtransmission tower

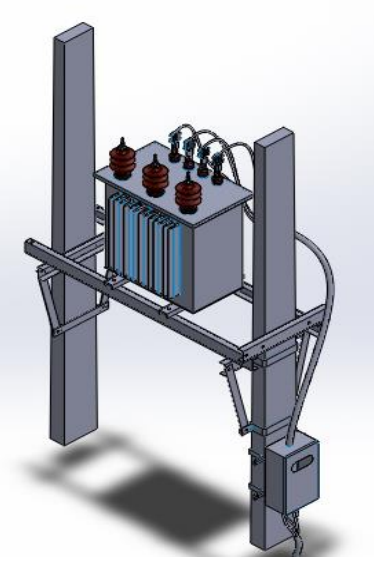

(b) Transformer

Fig. 5. CAD Design in SolidWorks

\subsection{Electrical diagram}

The realism and behavior of the electrical system is carried out with ge-nerada simulation and electrical variables delivered by the DigSilent Power Factory software, same that for its functionality the data of generator board and transformers as well as the electrical parameters of the transmission line, loads, circuit breakers and disconnectors must be inserted in the DigSilent editor according to real values of: active power, reactive power, resistance, impedance, type of conductor, length of conductor and other configurations. The feasibility of the software not only focuses on the creation of diagrams for power flows in the study of the effects on the distribution of loads when losses are generated, but also allows simulating phenomena of system failures, being directly affected by external overcurrents in different bars, the incidence of these overcurrents in the components and in the most critical case when this event occurs at the exact moment of a maintenance. The power flow diagrams and system faults are (shown in Fig. 6. and Fig. 7). 


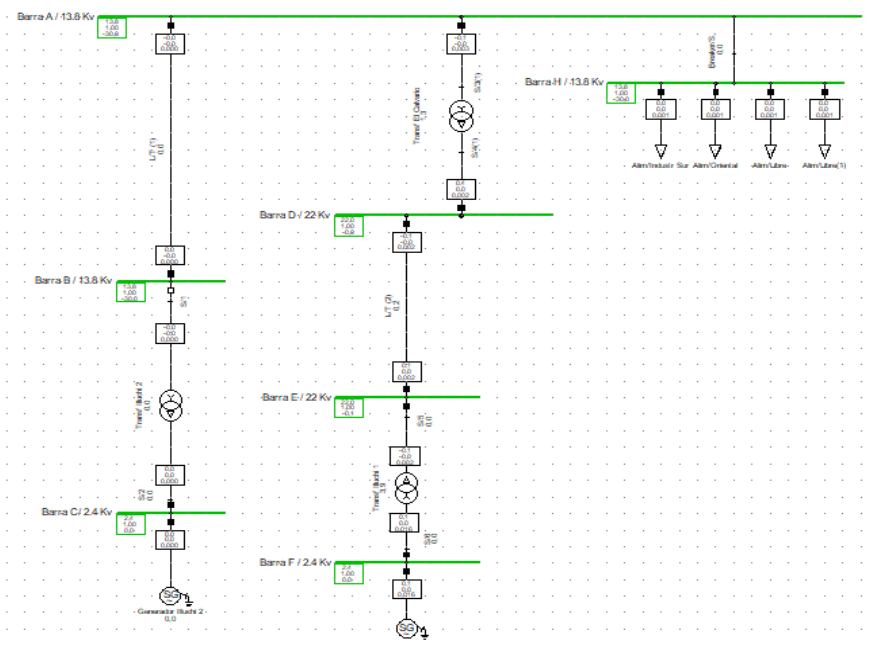

Fig. 6. System power flow in DigSilent Power Factory

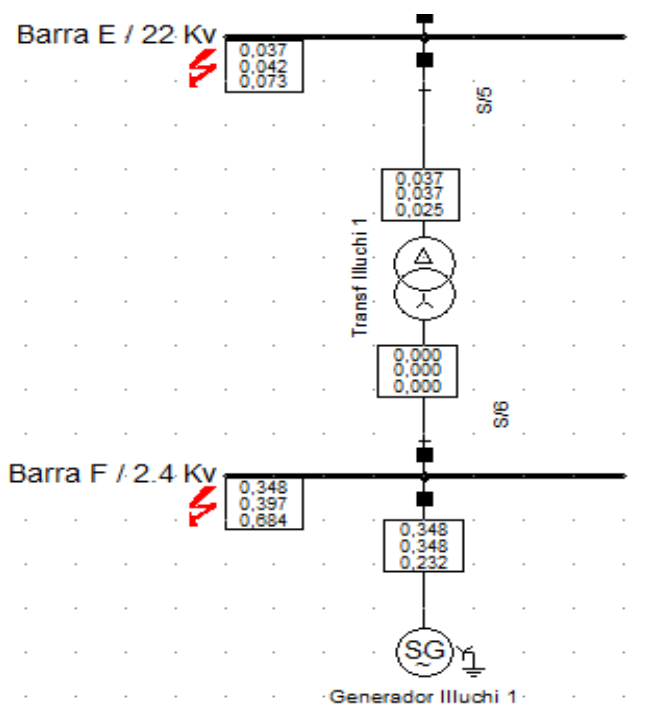

Fig. 7. Bar (E/F) failure at DigSilent Power Factory.

\subsection{Design of operators according to levels}

The information room has a menu that allows you to select from 4 levels of operators according to levels, in order to limit the activities and responsibilities of each member of the team by providing a learning method focused on each of the maintenance maneuvers and operation on energized lines. Avatars are created and customized in Adobe 
Fuse software. (See Fig. 8). Each one of them is created with their own personal protective equipment and safety accessories such as: shirt, work trousers, helmet, goggles and gloves.

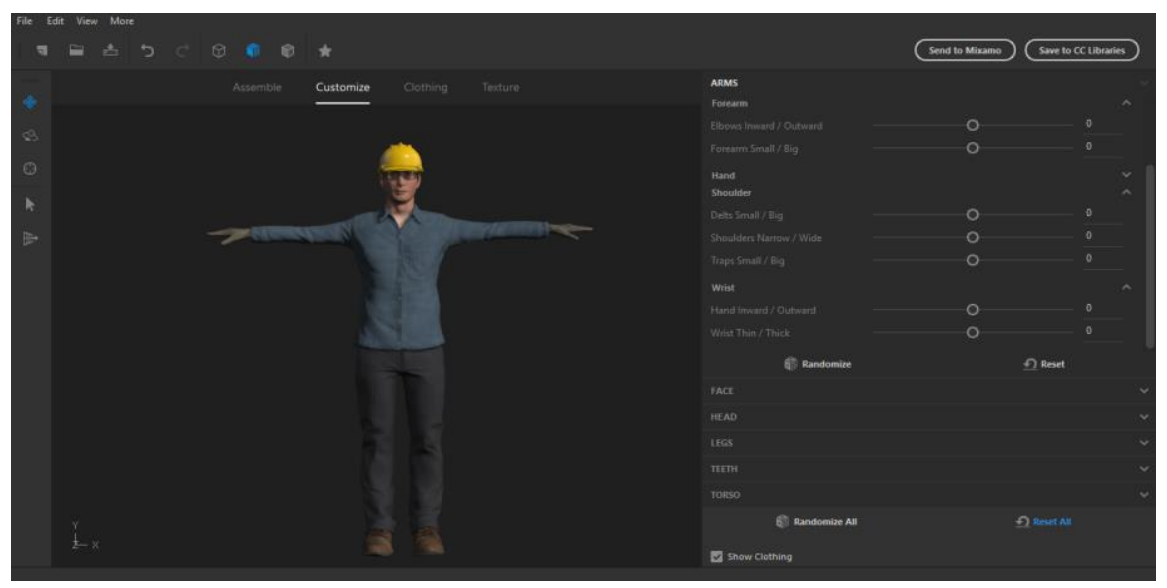

Fig. 8. Avatar created in Adobe Fuse

(Fig. 9). presents the levels of the operators: i) Chief of maintenance, EngineerMaster-High level; controls, coordinates with the group leader maintenance maneuvers and energized line operation and ensures compliance with safety standards; ii) Overseer, Engineer-High level; performs work orders and delivers materials according to the operations to be performed in the work area; iii) Chief of group, Technologist-half level; coordinates, receives materials according to work orders and performs maintenance maneuvers on energized lines; $i$ ) Operator, Low-level technician; fulfills work orders, performs operations and maneuvers such as: cleaning and change of insulators, change and relocation of crossheads, change of disconnectors, assembly and disassembly of transformer. 


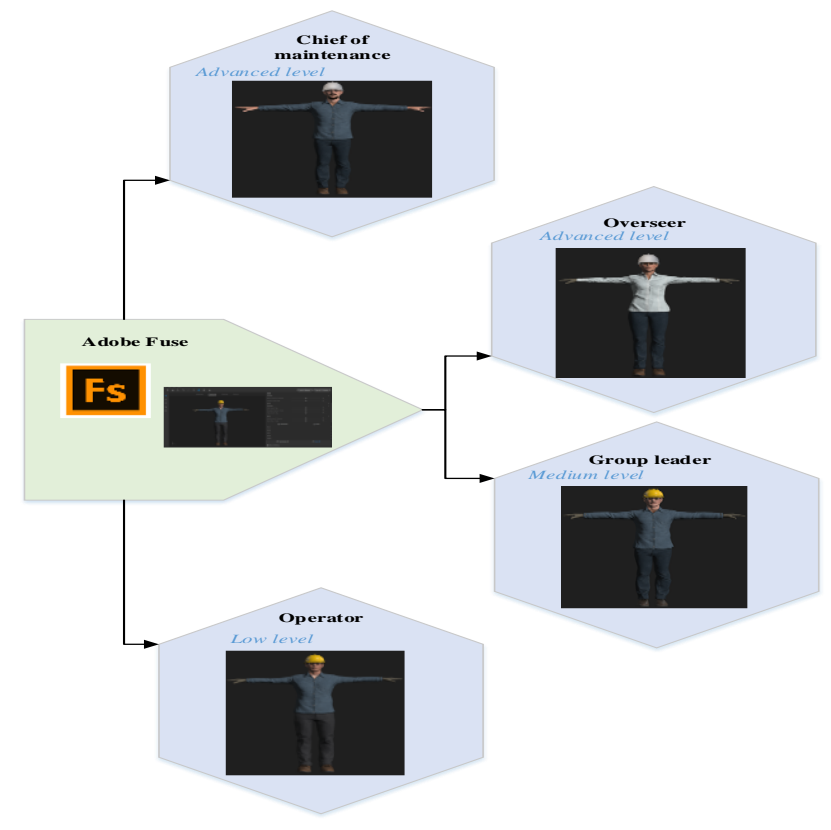

Fig. 9. Operator levels created in Adobe Fuse

\section{$4 \quad$ Multi-user System}

This section focuses on multi-user application development, from the selection of avatars in the environment according to established hierarchies to the interaction between operators in a collaborative environment.

(Fig. 10). shows the multi-user system, which complies with the client-server architecture, allowing the sending and receiving of messages by the operator (cliente) to the server which in turn replicates the information to the other operators by storing them in the database.

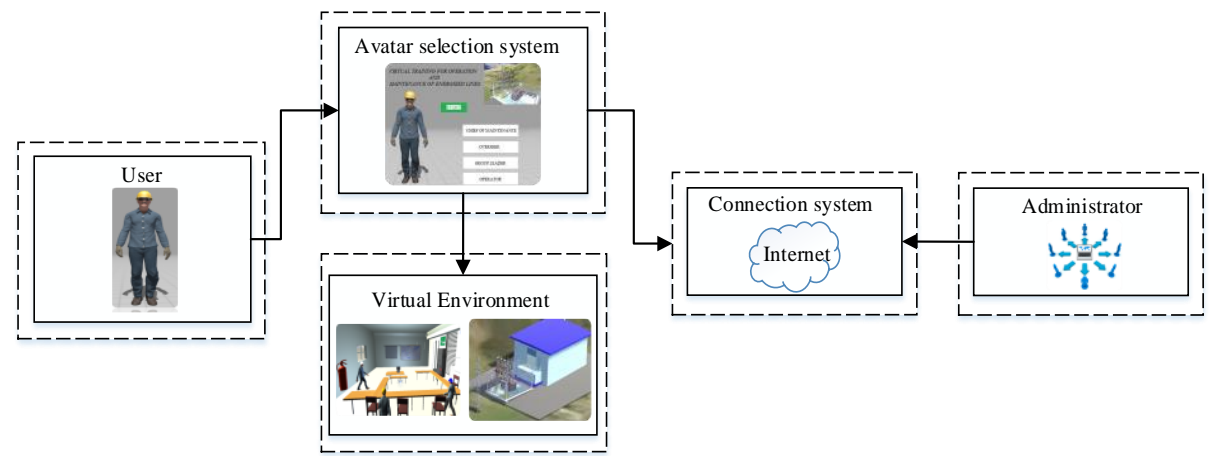

Fig. 10. Multi-user system 


\subsection{Maintenance operator selection}

Maintenance operator selection on energized lines depends on hierarchical levels, are mainly characterised by the colour of the helmet and the work clothes, that's why in the menu that starts the application you will find the positions of each avatar who represents the operators of the work team in energized lines, that when selecting the position will be registered with a name and ID that is unique for each operator.

(Fig. 11). presents the system of selection of the personnel according to the positions and responsibilities of the operators such as: i) Maintenance Manager, Engineer-Master-High level; ii) Overseer, Engineer-High level; iii) Group leader, Technologist- medium level; iv) Operator, Technical- low level.

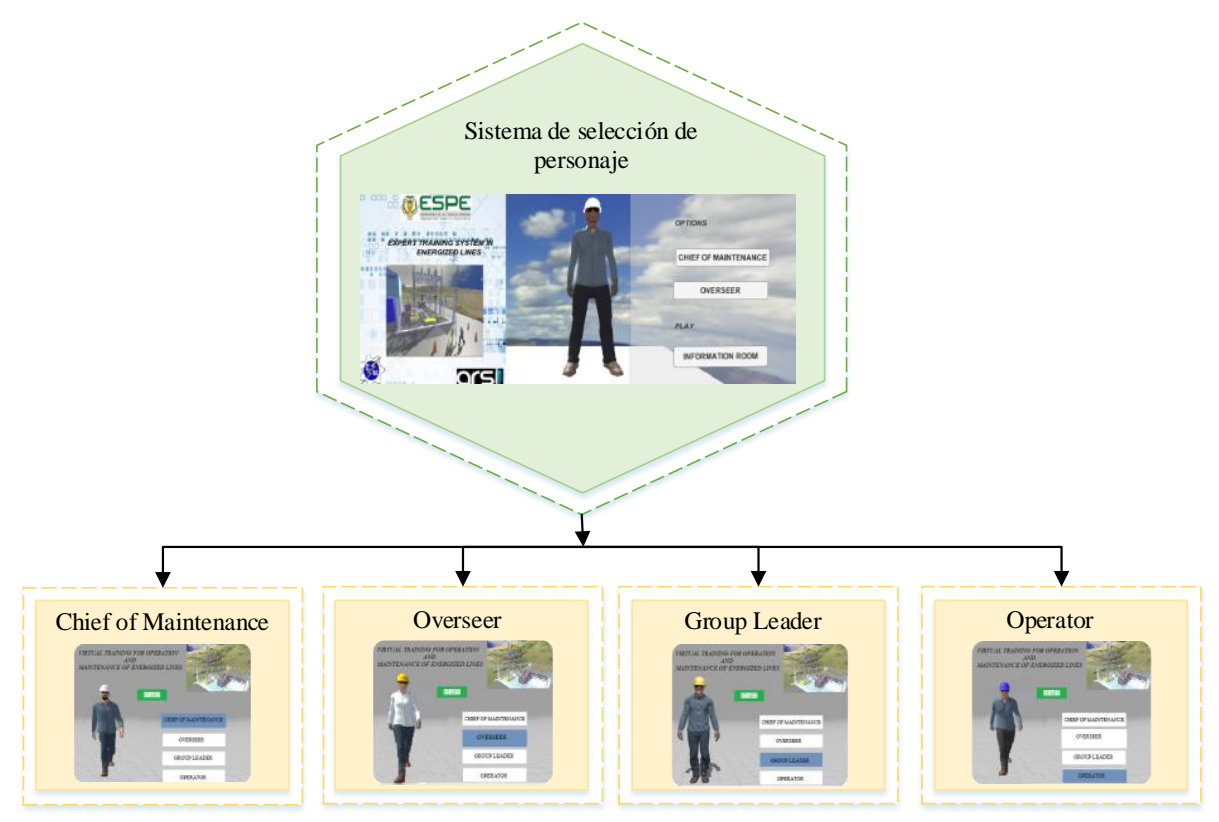

Fig. 11. Personnel selection system

\section{$5 \quad$ Results and Discussions}

The results achieved by the multi-user virtual training expert system are described in this section as an extra tool for learning and correct management in the operation and maintenance of energized lines, due to the dangerous events represented by such work and the high cost of line protection equipment, by means of the virtual application the novel advantage was obtained of simulating different failure events caused in the electrical power system, specifically on the main busbarswithout the need to directly expose operators who have integral experience theoretical-practical learning, focused on an 
efficient collaborative work between groups in case of emergencies caused in the system.

The virtual work environment shows two training modes (operator and visitor) that allow user interaction and immersion according to the learning requirements as (shown in Fig. 12).

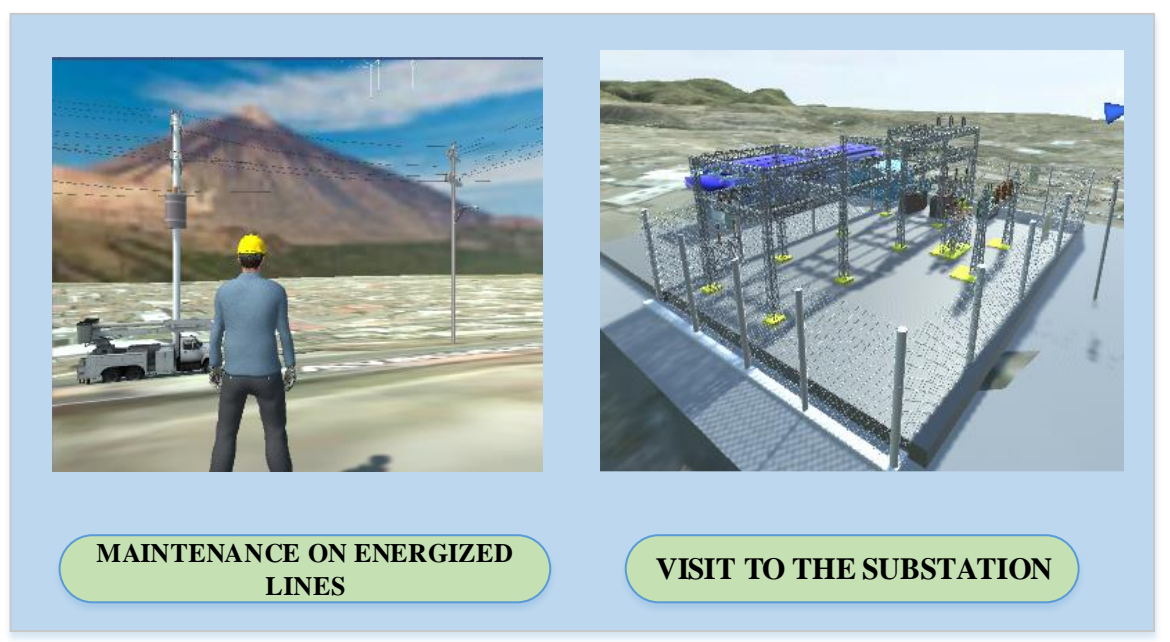

Fig. 12. Training mode menu

Maintenance mode on energized lines, In this mode the user from any location can access the environment by entering the name and id, through which it is presents a selection menu of maintenance operators by hierarchical position according to activities and responsibilities as (shown in Fig. 13). After selection the operator enters the information room in which visual and auditory instructions on safety standards, diagrams and maintenance protocols are displayed. (See Fig. 14).

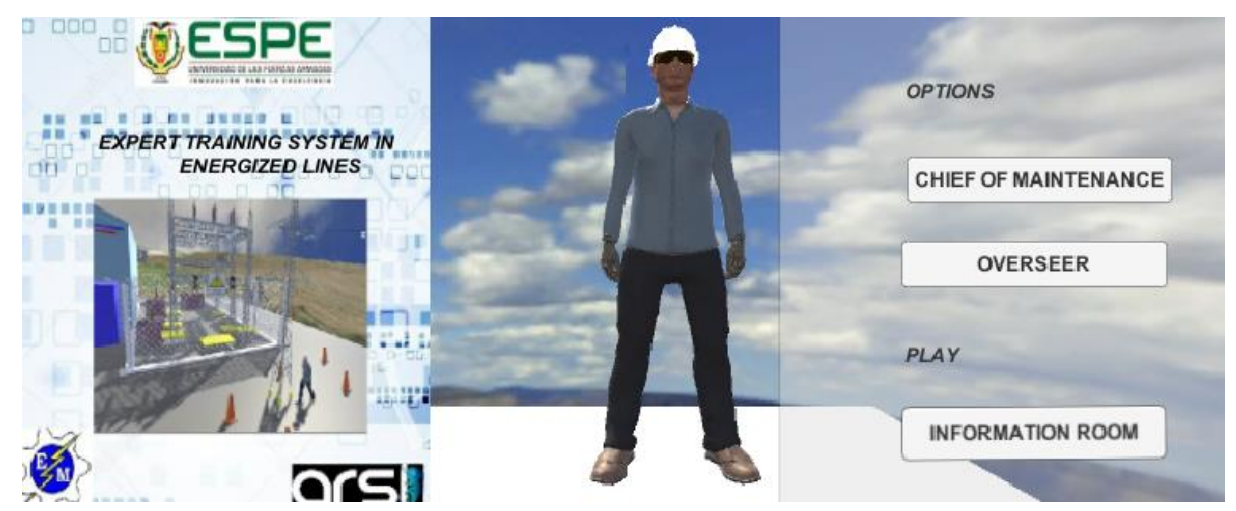

Fig. 13. Hierarchy selection menu 


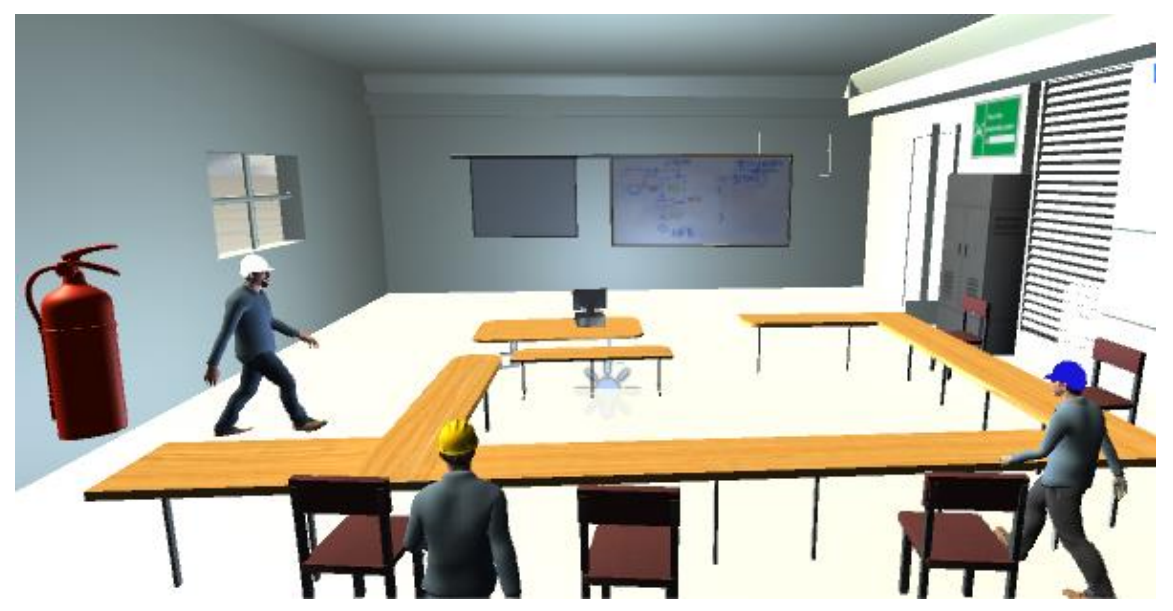

Fig. 14. Training and information room

Selection of maneuver and work area, A menu is presented to select the type of maintenance in the sub-transmission or distribution system with maneuvers such as: assembly and disassembly of single phase transformer, change and cleaning of insulators and change of crossarms. (See Fig. 15.)

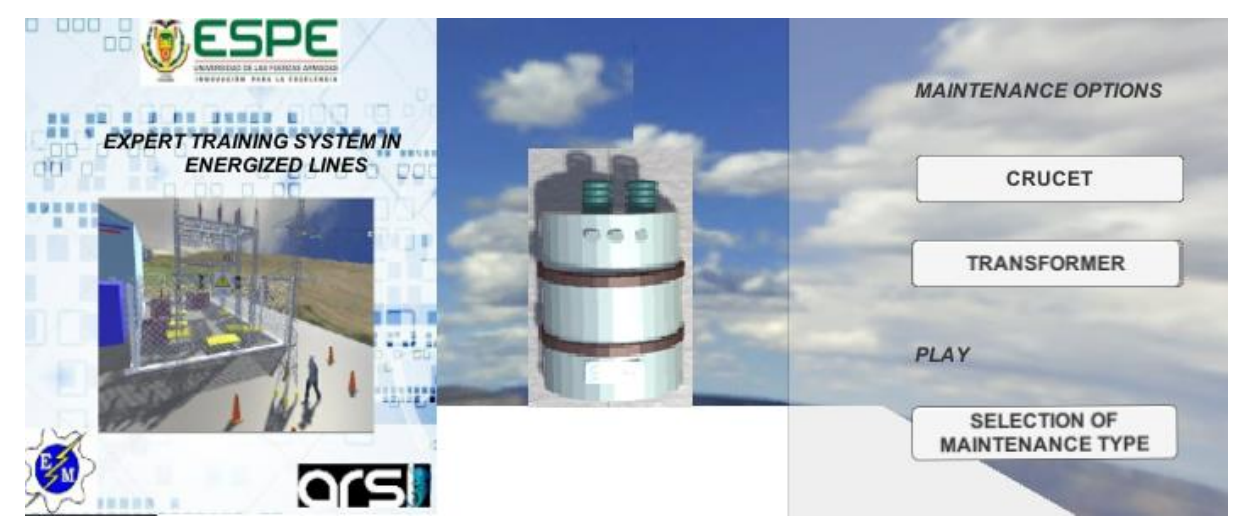

Fig. 15. Selection menu of the type of manoeuvre

Work area, In this environment the operator is able to visualize and interact directly with the potency system, substation of elevation, substation of reduction, insulators, lines of transmission, power transformer and other electromechanical components ( See Fig. 16), for perform maintenance maneuvers on lines energized under safety standards and protocols. 


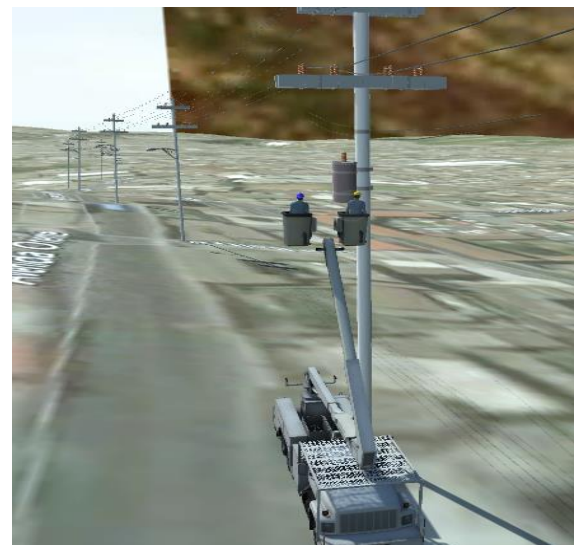

(a) Mantenance of transformer

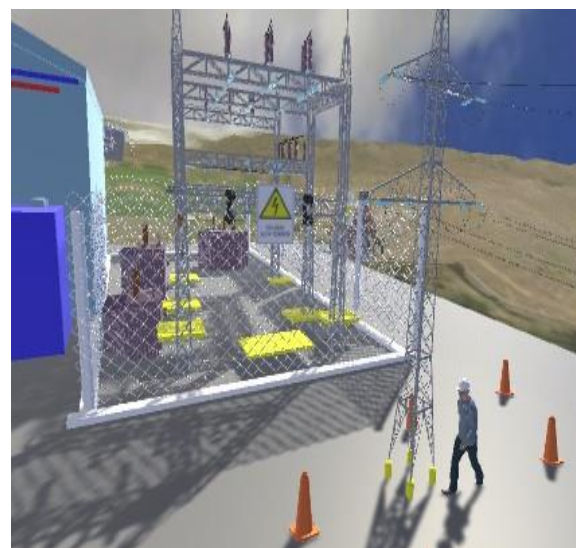

(b) Inspecction of médium voltaje lines Fig. 16. Operation and maintenance maneuver in medium voltage

Multi-user, The training system has the novel alternative for training operators on a multi-user platform, in which it allows the immersion and interaction of several users to the work area, according to the complexity of the manoeuvre, as (shown in the Fig. 17).

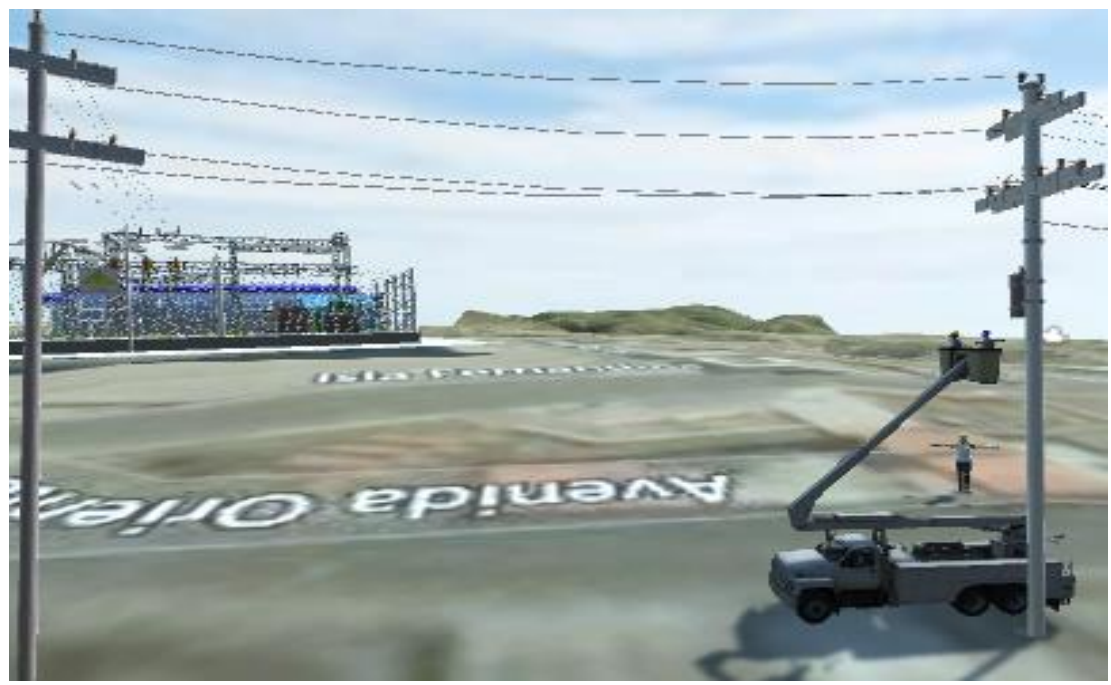

Fig. 17. Manoeuvre of collaborative maintenance 
Failt model, In (Fig. 18) is displayed the fault occurred in the E-bar of the system caused by an opening error in a disconnector provoking that the circuit opens and generates an electric arc as (shown in Fig. 19), and The switches of the Illuchi 1 lifting substation are be directly affected. The Programming facilitates the effect of visual and auditory realism at the exact moment of the electrical emergency.

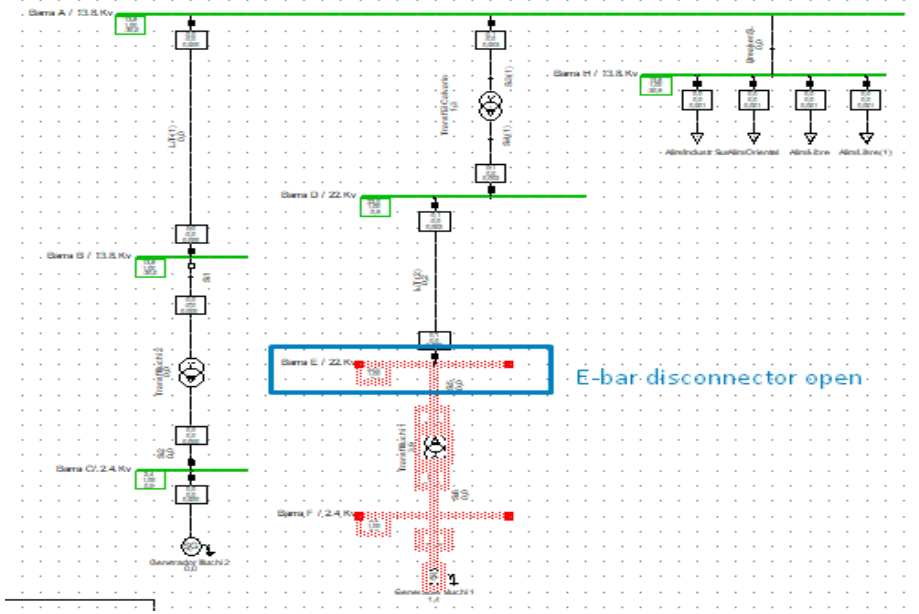

Fig. 18. E-bar disconnector open

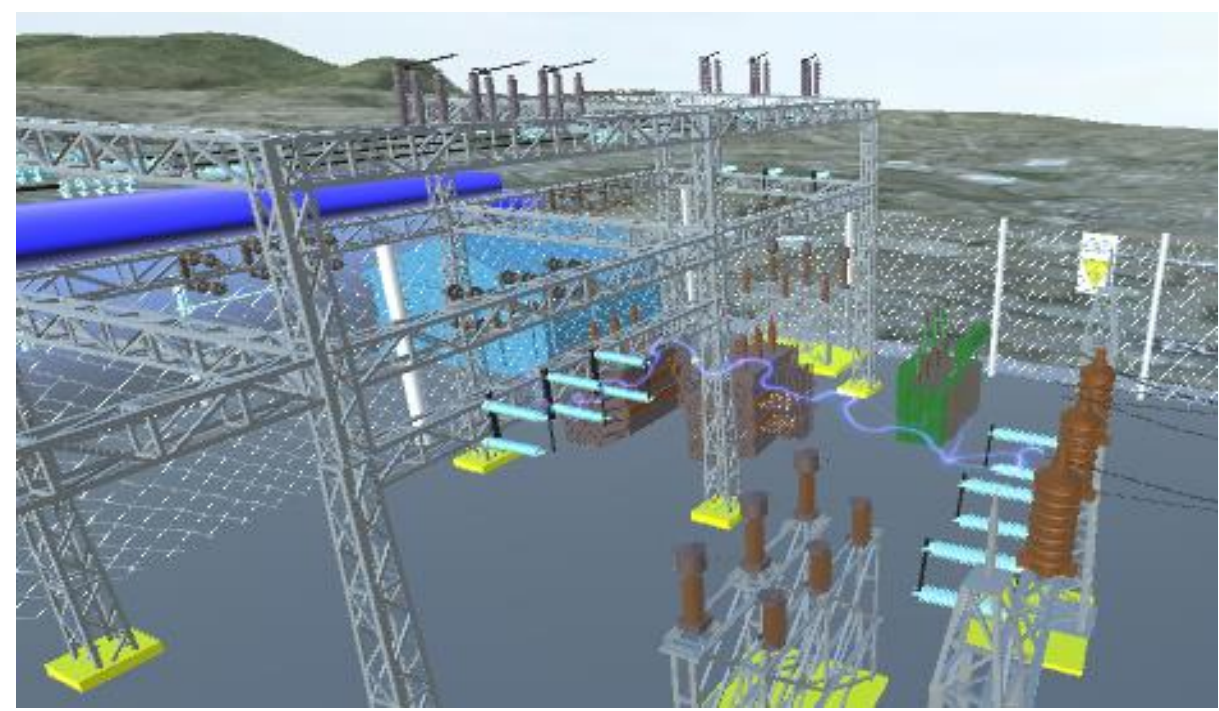

Fig. 19. Electric arc on E-bar

Visitor mode, In this mode you have access to the three substations Illuchi 1, Illuchi 2 and El Calvario in which students and new operators have the advantage of visiting and 
learning about the elements that make up the system through an audible guide that facilitates tour and learning in the electric field, (See Fig. 20).

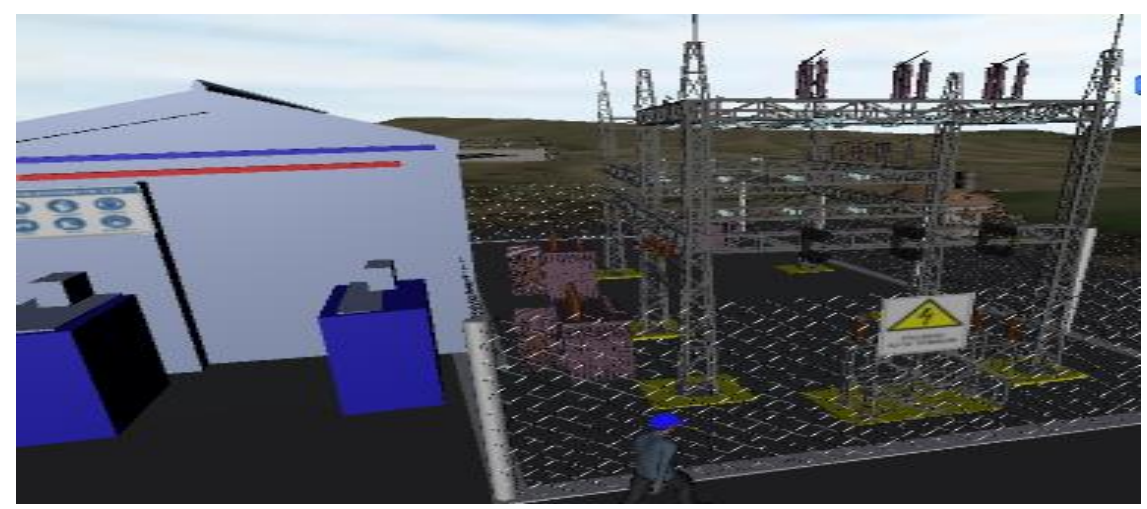

Fig. 20. Visit mode to El Calvario substation

The evaluation and validation of the multi-user training assistant provides a range of approaches to the effectiveness of training tasks and maintenance maneuvers. Where finally, the joint evaluation verifies the validity of the user-system interface in real situations, quantifying the results in terms of benefit (knowledge, cost and extra risk-free training tool). The SUS [17] usability of the expert system was evaluated by means of a questionnaire of questions. During the development of the evaluation a group of 15 people, among them 10 students and 5 professors of the Career of Electromechanical Engineering located in different locations: Research Laboratory, Network Laboratory \& Industrial Processes Control participated in this evaluation process, with students who take the subjects of Maintenance Engineering and Electrical Systems.(See Fig. 21). The evaluators issued criteria with respect to the animations and presentation of the maintenance procedures for the analysis of the information received by the evaluated and possible improvements in terms of redesign of the system. Subsequently, research was carried out whose main focus was the improvement of training through two groups of people: users trained using the conventional method (theoretical knowledge) versus users trained (theoretical knowledge plus training in the Virtual Reality environment), showing a positive influence on the improvement of cognitive skills, collaboration of students and teachers and retention of knowledge.

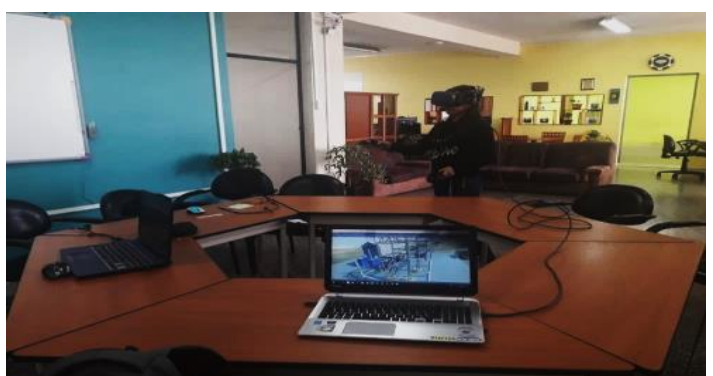

Fig. 21. Evaluation of the application with VR devices 
In the Table 1 . The items for the usability assessment corresponding to the application are shown.

Table 1. Evaluation results.

\begin{tabular}{ll}
\hline $\mathrm{N}^{\circ}-$ & QUESTIONS \\
\hline
\end{tabular}

1 Have you used the virtual reality devices: HTC and GEAR VR?

2 The assistant for energized lines training is easy to use and intuitive?

3

Does the virtual reality environment have all the elements of an electrical system for better familiarization?

4 Do you need the external help of a technician for the use of the application?

5

Can you easily perform the maintenance operations shown by the application?

6 Did the signage shown in the environment contribute to learning about safety issues?

7 Did the multi-user system allow you to interact collaboratively in different maintenance operations with several users at the same time?

8 Did the application help you improve your cognitive and collaborative skills?

9 Would you recommend the expert training system as an extra theoreticalpractical training tool that is taught in the educational field?

10

Is learning through virtual reality technologies new to be applied in education?

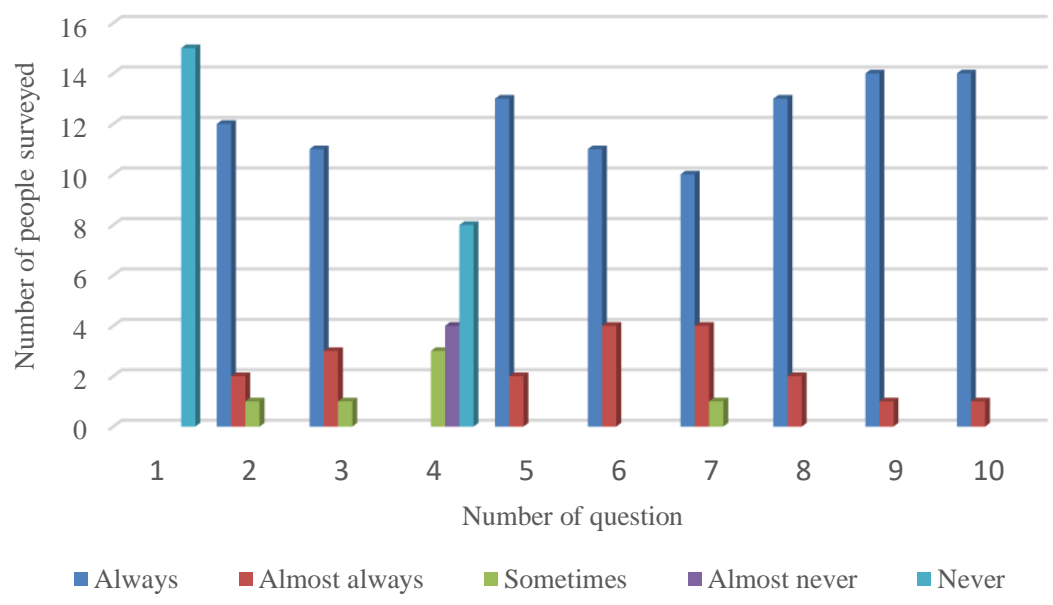

Fig. 22. Results of the evaluation of the virtual training system 
The results obtained are (shown in Figure 22), where 14 of the 15 people evaluated indicate that the virtual application for training operators on energized lines contributes to the learning and development of the cognitive skills of students and teachers in the area, since it is a novel tool that is easily accessible as a theoretical-practical support when interacting with the components of the electrical power system.

\section{Conclusions}

The article presents the development of the application of the multi-user training system based on virtual reality, within the electrical area in which it offers an assisted followup to the user within the recognition of processes and electrical elements of subtransmission and distribution contributing to the education and training of professionals by means of immersion and interaction of operation and maintenance manoeuvres in a risk-free environment optimizing economic resources, time and infrastructure.

The Unity 3D graphics engine features an easy-to-use interface that provides the realism of an electrical power system through interaction between multi-user system operators whose experimental results show the development of skills and abilities in a collaborative environment, renewing current training models.

Sending data from Unity 3D electrical software and graphics engine provides analysis of possible faults which can be critically caused by bad maneuvers or external conditions in the electrical power system (substation bars, distribution systems). The objective of the development of this application is to provide an extra training tool that facilitates learning the maneuvers and safety protocols in the operation and maintenance of energized lines.

The virtual environment is currently used by professors and students of the career of engineering in Electromechanics at the University of the Armed Forces ESPE that facilitates your theoretical-practical training, this training system based on the immersive virtual reality system can be easily installed on a computer and through the Internet interact with the multi-user system and strengthen collaborative work.

In the future it is desired to integrate an augmented reality system that facilitates maintenance not only in energized lines but also in electrical substations, starting out like this from his generation.

\section{References}

1. M. Faccio, A. Persona, F. Sgarbossa y G. \& Zanin, «Industrial maintenance policy development: A quantitative framework.,» International Journal of Production Economics, vol. 147, pp. 85-93, 2014.

2. R. Van de Kerkhof, H. Akkermans y N. \& Noorderhaven, « Knowledge lost in data: organizational impediments to condition-based maintenance in the process industry.,» Logistics and Supply Chain Innovation Springer, Cham., pp. 223-237, 2016. 
3. J. Daily y J. \& Peterson, «Predictive maintenance: How big data analysis can improve maintenance.,,$n$ Supply Chain Integration Challenges in Commercial Aerospace Springer, Cham., pp. 267-278, 2017.

4. A. Koksal y A. \& Ozdemir, «mproved transformer maintenance plan for reliability centred asset management of power transmission system.,» IET Generation, Transmission \& Distribution, vol. X, $\mathrm{n}^{\circ}$ 8, pp. 1976-1983, 2017.

5. C. Barbosa y F. \& Nallin, «Corrosion detection robot for energized power lines,» In Proceedings of the 2014 3rd International Conference on Applied Robotics for the Power Industry IEEE., pp. 1-6, 2014.

6. D. K. Neitzel, «Electrical safety when working near overhead power lines.,» $n$ 2016 IEEE PES 13th International Conference on Transmission \& Distribution Construction, Operation \& Live-Line Maintenance (ESMO) IEEE, pp. 1-5, 2016.

7. I. Galvan, A. Ayala, E. Rodríguez y G. \& Arroyo, «Virtual reality training system for maintenance of underground lines in power distribution system,» Virtual Reality, 2016.

8. A. Ayala, I. Galván, G. Pérez, M. Ramirez y J. \& Muñoz, «Virtual reality training system for maintenance and operation of high-voltage overhead power lines,» Third International Conference on Innovative Computing Tecnology (INTECH 2013), 2013.

9. M. Perez, G. Arroyo y A. \& Ayala, «Perez-Ramirez, M., Arroyo-Figueroa, G., $\&$ Ayala, A. (2019). The use of a virtual reality training system to improve technical skill in the maintenance of live-line power distribution networks,» Interactive Learning Environments, pp. 1-18, 2016.

10. ZayasB. y M. \& Perez, «An instructional design model for virtual reality training environments,» In EdMedia+ Innovate Learning. Association for the Advancement of Computing in Education (AACE)., pp. 483-488, 2015.

11. B. Li, Y. Bi, Q. He, J. Ren y Z. \& Li, «A low-complexity method for authoring an interactive virtual maintenance training system of hydroelectric generating equipment,» Computers in Industry, pp. 159-172, 2018.

12. Y. Hernández, M. Pérez, W. Ramírez, E. Ayala y N. \& Ontiveros, «Architecture of an Intelligent Training System based on Virtual Environments for Electricity Distribution Substations,» Research in Computing science, pp. 63-70, 2016.

13. P. Dos Reis, C. Matos, P. Diniz, D. Silva, W. Dantas, G. Braz y A. \& Araújo, «An Immersive Virtual Reality Application for Collaborative Training of Power Systems Operators,» In 2015 XVII Symposium on Virtual and Augmented Reality IEEE, pp. 121-126, 2015.

14. M. Chiluisa, R. Mullo y V. H. \& Andaluz, «Training in Virtual Environments for Hybrid Power Plant,» In International Symposium on Visual Computing. Springer, Cham., pp. 193-204, 2018.

15. S. Zhang, S. Ying, Y. Shao, W. Gao, Y. Liang, P. Peng y X. \& Luo, «Design and Application of Electric Power Skill Training Platform Based on Virtual Reality Technology,» In 2018 Chinese Automation Congress (CAC). IEEE, pp. 1548-1551, 2018. 
16. A. Cardoso, I. do Santos Peres, E. Lamounier, G. Lima, M. Miranda y I. \& Moraes, «Associating Holography Techniques with BIM Practices for Electrical Substation Design,» In International Conference on Applied Human Factors and Ergonomics. Springer, Cham., pp. 37-47, 2017.

17. C. M. L. Z. a. L. H. Linkin, «Modeling risk behaviors in virtual environment based on Multi-agent,» 2010 The 2nd Internationla Conference on Computer and Automation Engineering (ICCAE), 2010. 